\title{
Shifts of Attention During Spatial Language Comprehension A Computational Investigation
}

\author{
Thomas Kluth $^{1}$, Michele Burigo ${ }^{1}$, and Pia Knoeferle ${ }^{2}$ \\ ${ }^{1}$ Language \& Cognition Group, CITEC (Cognitive Interaction Technology Excellence Cluster), Bielefeld University, \\ Inspiration 1, 33619 Bielefeld, Germany \\ ${ }^{2}$ Department of German Language and Linguistics, Humboldt University, Unter den Linden 6, 10099 Berlin, Germany \\ \{tkluth,mburigo\}@cit-ec.uni-bielefeld.de,pia.knoeferle@hu-berlin.de
}

Keywords: Spatial Language, Spatial Relations, Cognitive Modeling, Visual Attention.

Abstract: Regier and Carlson (2001) have investigated the processing of spatial prepositions and developed a cognitive model that formalizes how spatial prepositions are evaluated against depicted spatial relations between objects. In their Attentional Vector Sum (AVS) model, a population of vectors is weighted with visual attention, rooted at the reference object and pointing to the located object. The deviation of the vector sum from a reference direction is then used to evaluate the goodness-of-fit of the spatial preposition. Crucially, the AVS model assumes a shift of attention from the reference object to the located object. The direction of this shift has been challenged by recent psycholinguistic and neuroscientific findings. We propose a modified version of the AVS model (the rAVS model) that integrates these findings. In the rAVS model, attention shifts from the located object to the reference object in contrast to the attentional shift from the reference object to the located object implemented in the AVS model. Our model simulations show that the rAVS model accounts for both the data that inspired the AVS model and the most recent findings. ${ }^{a}$

\footnotetext{
${ }^{a}$ This work was presented at the 8th International Conference on Agents and Artificial Intelligence 2016 (ICAART 2016) that took place in Rome, Italy, from February 24 to 26, 2016 (http: / www. icaart.org). Unfortunately, in the version of this paper published in the conference proceedings the citations are messed up. This version is easier to read.
}

\section{INTRODUCTION}

Imagine a household robot that helps you in the kitchen. You might want the robot to pass you the salt and instruct it as follows: "Could you pass me the salt? It is to the left of the stove". Here, the salt is the located object (LO), because it should be located relative to the reference object (RO, the stove). To find the salt, the robot should interpret this sentence the way you meant it. In the interaction with artificial systems, humans often need to instruct artificial systems to interact with objects in their environment. To this end, artificial systems need to interpret spatial language, i.e., language that describes the locations of the objects of interest. To make the interaction as natural as possible, artificial systems should understand spatial language the way humans do it. The implementation of psychologically validated computational models of spatial language into artificial systems might thus prove useful. With these kind of models, artificial systems could interpret and generate human-like spatial language.

Logan and Sadler (1996) were the first to outline a computational framework of the processes that are assumed to take place when humans understand spatial language. Their framework consists of "four different kinds of processes: spatial indexing, reference frame adjustment, spatial template alignment, and computing goodness of fit" (Logan \& Sadler, 1996, p. 500).

Spatial indexing is required to bound the perceptual representations of the $\mathrm{RO}$ and the $\mathrm{LO}$ to their corresponding conceptual representations. According to Logan and Sadler (1996, p. 499), "the viewer's attention should move from the reference object to the located object". Reference frame adjustment consists of imposing a reference frame on the RO and setting its parameters (origin, orientation, direction, scale). "The reference frame is a three-dimensional coordinate system [...]" (Logan \& Sadler, 1996, p. 499). Spatial template alignment is the process of imposing a spatial template on the RO that is aligned with the reference frame. A spatial template consists of re- 
gions of acceptability of a spatial relation. Every spatial relation is theorized to have its own spatial template. Finally, computing goodness of fit is the evaluation of the location of the LO in the aligned spatial template.

Trying to identify possible nonlinguistic mechanisms that underlie the rating of spatial prepositions, Regier and Carlson (2001) developed a cognitive model: the Attentional Vector Sum (AVS) model. ${ }^{1}$ This model - based on the assumption that goodnessof-fit ratings for spatial prepositions against depicted objects reflect language processing - accounts for a range of empirical findings in spatial language processing. A central mechanism in the AVS model concerns the role of attention for the understanding of spatial relations.

Direction of the Attentional Shift Previous research has shown that visual attention is needed to process spatial relations (Logan, 1994, 1995; Logan \& Sadler, 1996; see Carlson \& Logan, 2005 for a review). The AVS model has formalized the role of visual attention. Although Regier and Carlson (2001) do not explicitly talk about attentional shifts, the AVS model can be interpreted as assuming a shift of attention from the RO to the LO. Regier and Carlson (2001) motivate the implementation of attention based on studies conducted by Logan (1994) and Logan (1995, p. 115): "The linguistic distinction between located and reference objects specifies a direction for attention to move - from the reference object to the located object." (See also Logan \& Sadler, 1996, p. 499: "the viewer's attention should move from the reference object to the located object"). But are humans actually shifting their attention in this direction while they are understanding a spatial preposition?

Evidence for shifts of covert attention is provided by studies in the field of cognitive neuroscience by Franconeri and colleagues (Franconeri, Scimeca, Roth, Helseth, \& Kahn, 2012; Roth \& Franconeri, 2012). Using EEG, Franconeri et al. (2012) showed that humans shift their covert attention when they process spatial relations. In their first experiment, they presented four objects of which two had the same shape but different colors. Two objects were placed to the right and two objects were placed to the left of a fixation cross such that two different shapes appeared on each side of the cross. Participants had to

\footnotetext{
${ }^{1}$ Apart from the AVS model, a range of other computational models of spatial language processing were also proposed (e.g., Cangelosi et al., 2005; Gapp, 1995; Kelleher, Kruijff, \& Costello, 2006; Richter, Lins, Schneegans, Sandamirskaya, \& Schöner, 2014).
}

fixate the fixation cross and judge whether, say, the orange circle was left or right of the cyan circle. After the stimulus display was shown, participants chose one spatial relation out of two possible arrangements on a response screen (cyan circle left of orange circle or orange circle left of cyan circle). During the experiment, event-related potentials were recorded. All experiments reported in Franconeri et al. (2012) revealed that participants shifted their attention from one object to the other object, although they had been instructed to attend to both objects simultaneously. However, the role of the direction of these shifts remained unclear in Franconeri et al. (2012).

In another experiment, Roth and Franconeri (2012) presented questions like "Is red left of green?" to participants. Subsequently, either a red or a green object appeared on the screen, followed shortly afterwards $(0-233 \mathrm{~ms})$ by a green or a red object respectively. By manipulating the presentation order of the objects, a shift of attention was cued. Participants were faster to verify the question if the presentation order was the same as the order in the question. Roth and Franconeri (2012) interpreted this as evidence that the perceptual representation of a spatial relation follows its linguistic representation.

Evidence that a shift of attention from the RO to the LO as suggested in the AVS model is not necessary for understanding spatial language has been recently reported by Burigo and Knoeferle (2015), who conducted a visual world study. Here, participants inspected a display and listened to spoken utterances while their eye movements were recorded. Note that Burigo and Knoeferle (2015) investigated overt attention - unlike Franconeri et al. (2012) and Roth and Franconeri (2012) who studied covert attention. Burigo and Knoeferle (2015) presented sentences with two German spatial prepositions (über [above] and unter [below]) across four different tasks. The RO and the LO of the sentence as well as a competitor object (not mentioned in the sentence) were presented on a computer screen. In their first experiment, participants verified the spatial sentence as quickly as possible, even before the sentence ended. In their second experiment, participants also verified the sentence, but they had to wait until the sentence was over. The third experiment consisted of a passive listening task, i.e., no response was required from the participants. Finally, in the fourth experiment, a gaze-contingent trigger was used: the competitor object and either the LO or the RO was removed from the display after participants had inspected the LO at least once.

The results from this study revealed that participants shifted their overt attention from the RO to the 
LO, as predicted by the AVS model. However, the task modulated the presence of these shifts. These shifts were only frequent in the post-sentence verification experiment (experiment 2), but infrequent in the other experiments. Crucially, if participants did not shift their attention from the RO to the LO, they performed equally well (as accuracy was not affected) - i.e., they were able to understand the sentence without shifting their attention from the RO to the LO.

By contrast, participants frequently shifted gaze overtly from the LO towards the RO (in line with the incremental interpretation of the spoken sentence). This suggested that people may be able to apprehend a spatial relation with an overt attentional shift from the LO to the RO (and not from the RO to the LO as suggested by the AVS model).

Thus, the direction of the attentional shift as implemented in the AVS model conflicts with recent empirical findings. We propose a modified version of the AVS model: the reversed AVS (rAVS) model, where the attentional shift has been reversed. Instead of a shift from the RO to the LO, we implemented a shift from the LO to the RO. We designed the rAVS model otherwise to be as similar as possible to the AVS model. By doing so, we can compare the influence of the reversed shift on the performance of the two models.

\section{THE MODELS}

In this section, we first describe the AVS model, since the proposed rAVS model is based on the structure of the AVS model and modifies some parts of it. Next, we introduce the rAVS model.

\subsection{The AVS Model}

Regier and Carlson (2001) proposed a cognitive model of spatial term comprehension: the Attentional Vector Sum (AVS) model. The AVS model takes the 2D-location and the 2D-shape of a RO, the 2Dlocation of a LO, and a spatial preposition as input and computes an acceptability rating (i.e., how well the preposition describes the location of the LO relative to the RO). In the following, we are presenting how the AVS model processes the spatial relation between the RO and the LO and how it computes the acceptability rating. The AVS model consists of two components: The angular component and the height component. Figures 1(a)-1(c) depict the angular component which we describe first. Figure 1(d) visualizes the height component that we describe thereafter.

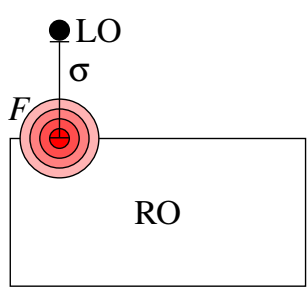

(a) Attentional Distribution. Darker color means higher amount of attention.

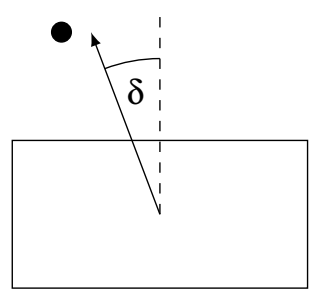

(c) Deviation of the final vector from canonical upright. The final vector does not necessarily point to the LO, because it is the weighted sum of all vectors in Figure 1(b).

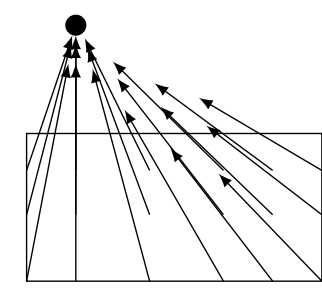

(b) Vectors weighted with attention and pointing to the LO.

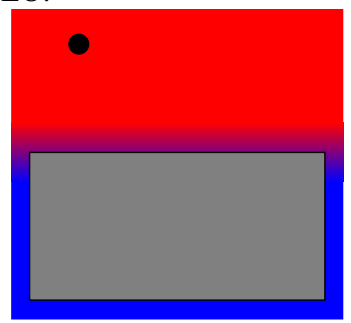

(d) Height Component. Red means a value of 1 and blue means a value of 0 . The height component modulates the outcome of the angular component with respect to the y-coordinate of the LO: A 0 results in a low rating, a 1 does not change the output of the angular component.

Figure 1: Schematized steps of the AVS model developed by Regier and Carlson (2001).

Angular component First, the AVS model defines the focus $F$ of a distribution of visual attention as the point on top of the RO "that is vertically aligned with the trajector [LO] or closest to being so aligned"2 (Regier \& Carlson, 2001, p. 277). Next, the model defines the distribution of attention on every point $i$ of the RO as follows (see Figure 1(a) for visualization):

$$
a_{i}=\exp \left(\frac{-d_{i}}{\lambda \cdot \sigma}\right)
$$

Here, $d_{i}$ is the euclidean distance between RO point $i$ and the attentional focus $F, \sigma$ is the euclidean distance between the attentional focus $F$ and the LO, and $\lambda$ is a free parameter. The resulting distribution of attention is highest at the focal point $F$ and declines exponentially with greater distance from F (see Figure 1(a)). Furthermore, the distance $\sigma$ of the LO to the RO as well as the free parameter $\lambda$ affect the width of the attentional distribution: A close LO results in a

\footnotetext{
${ }^{2}$ In the case of other prepositions, the corresponding part of the RO is chosen for the location of the focus (e.g., the focus lies on the bottom of the RO for below).
} 
more focused attentional distribution (a large decline of attention from point F) whereas a distant LO results in a more broad attentional distribution (a small decline of attention from point $F$ ).

In the next step, vectors $v_{i}$ are rooted at every point $i$ of the RO. All vectors are pointing to the LO and are weighted with the amount of attention $a_{i}$ that was previously defined (see Figure 1(b)). All these vectors are summed up to obtain a final vector:

$$
\overrightarrow{\text { direction }}=\sum_{i \in R O} a_{i} \cdot \vec{v}_{i}
$$

The deviation $\delta$ of this final vector to canonical upright (in the case of above) is measured (see Figure 1(c)) and used to obtain a rating with the help of the linear function $\mathrm{g}(\delta)$ that maps high deviations to low ratings and low deviations to high ratings:

$$
\mathrm{g}(\delta)=\text { slope } \cdot \delta+\text { intercept }
$$

Both, slope and intercept, are free parameters and $\delta$ is the angle between the sum of the vectors and canonical upright (in the case of above):

$$
\delta=\angle(\overrightarrow{\text { direction }}, \text { upright })
$$

Height Component $g(\delta)$ is the last step of the angular component. This value is then multiplied with the height component. The height component weights the angular component with the elevation of the LO relative to the top of the RO. It is defined as follows:

$$
\begin{gathered}
\operatorname{height}\left(y_{L O}\right)= \\
\frac{\operatorname{sig}\left(y_{L O}-\text { hightop, highgain }\right)+\operatorname{sig}\left(y_{L O}-\text { lowtop }, 1\right)}{2}
\end{gathered}
$$

Here, highgain is a free parameter, hightop (or lowtop) is the highest y-coordinate of the highest (or lowest) point on top of the $\mathrm{RO}$, and the $\operatorname{sig}(\cdot, \cdot)$ function is defined as:

$$
\operatorname{sig}(x, \text { gain })=\frac{1}{1+\exp (\operatorname{gain} \cdot(-x))}
$$

The AVS model has four free parameters in total: $\lambda$, slope, intercept, highgain. Taken together, the final acceptability rating is computed by the AVS model with the following formula:

$$
\operatorname{above}(L O, R O)=\mathrm{g}(\boldsymbol{\delta}) \cdot \operatorname{height}\left(y_{L O}\right)
$$

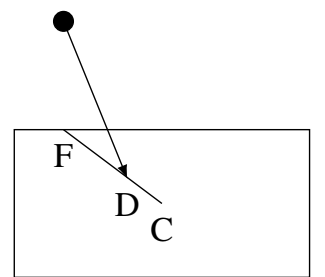

(a) Vector destination lies always on the line that connects $\mathrm{C}$ and $\mathrm{F}$.

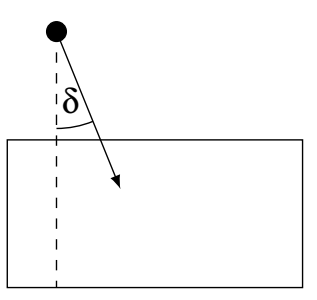

(c) Deviation from canonical downwards.

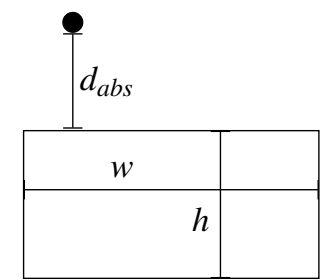

(b) Relative distance: Absolute distance divided by the size of the RO.

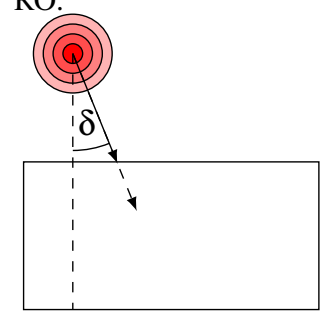

(d) The attentional distribution does not change the deviation.
Figure 2: Schematized steps of the rAVS model.

\subsection{The rAVS Model}

Although Regier and Carlson (2001) do not explicitly mention shifts of attention, the AVS model can be interpreted as assuming a shift of attention from the RO to the LO: This shift is implemented by the location of the attentional focus and in particular by the direction of the vectors (see Figs. 1(a)- 1(c)). As discussed before, this direction of the attentional shift conflicts with recent empirical findings (Burigo \& Knoeferle, 2015; Franconeri et al., 2012; Roth \& Franconeri, 2012). This is why our modified version of the AVS model, the reversed AVS (rAVS) model, implements a shift from the LO to the RO.

To this end, the rAVS model reverses the direction of the vectors in the vector sum in the following way: Instead of pointing from every point in the RO to the $\mathrm{LO}$, the vectors are pointing from every point in the LO to the RO. Since the LO is simplified as a single point in the AVS model, the vector sum in the rAVS model consists of only one vector. The end point of this vector, however, must be defined, since the RO has a mass.

In the rAVS model, the vector end point $D$ lies on the line between the center-of-mass $C$ of the RO and the proximal point $F$ (see Figure 2(a)). Here, $F$ is the same point as the attentional focus in the AVS model. Depending on the relative distance of the LO, the vector end point $D$ is closer to $C$ (for distant LOs) or closer to $F$ (for close LOs). Thus, the center-ofmass orientation is more important for distant LOs, 
whereas the proximal orientation becomes important for close LOs, which corresponds to the rating pattern found by Regier and Carlson (2001, experiment 7). The width of the attentional distribution in the AVS model has a similar effect.

In the rAVS model, the distance of a LO is considered in relative terms, i.e., the width and height of the $\mathrm{RO}$ change the relative distance of a LO, even if the absolute distance remains the same (see Figure 2(b)). The relative distance is computed as follows:

$$
d_{\text {rel. }}(L O, R O)=\frac{|L O, P|_{x}}{R O_{\text {width }}}+\frac{|L O, P|_{y}}{R O_{\text {height }}}
$$

Here, $P$ is the proximal point in the intuitive sense: The point on the RO that has the smallest absolute distance to the LO. $F$ is guaranteed to lie on top of the RO, whereas $P$ can also be at the left, right, or bottom of the RO. If $P$ is on top of the RO, $P$ equals $F$.

Furthermore, the computation of the vector end point $D$ is guided with an additional free parameter $\alpha$ (with $\alpha \geq 0$ ). The new parameter $\alpha$ and the relative distance interact within a linear function to obtain the new vector destination $D$. Here is the corresponding formula:

$$
\begin{gathered}
D= \\
\begin{cases}\overrightarrow{L O, C}+\left(-\alpha \cdot d_{r e l .}+1\right) \cdot \overrightarrow{C F} & \text { if }\left(-\alpha \cdot d_{r e l .}+1\right)>0 \\
C & \text { else }\end{cases}
\end{gathered}
$$

The direction of the vector $\overrightarrow{L O, D}$ is finally compared to canonical downwards instead of canonical upright (in the case of above, see Figure 2(c)) - similar to the angular component of the AVS model:

$$
\delta=\angle(\overrightarrow{L O, D}, \text { downwards })
$$

As in the AVS model, this angular deviation is then used as input for the linear function $g(\delta)$ (see equation 3) to obtain a value for the angular component. Note that a comparison to downwards is modeled, although the preposition is above. Roth and Franconeri $(2012$, p. 7) also mention this "counterintuitive, but certainly not computationally difficult" flip of the reference direction in their account.

In the rAVS model, the attentional focus lies on the LO. In fact, however, the location of the attentional focus as well as the attentional distribution do not matter for the rAVS model, because its weighted vector sum consists of only one single vector (due to the simplified LO). Since the length of the vector sum is not considered in the computation of the angle (neither in the AVS nor in the rAVS model), the amount of attention at the vector root is not of any importance for the final rating (as long as it is greater than zero, see Figure 2(d)). ${ }^{3}$

The height component of the AVS model is not changed in the rAVS model. So, it still takes the $y$ value of the LO as input and computes the height according to the grazing line of the RO (see equation 5). The final rating is obtained by multiplying the height component with the angular component:

$$
\operatorname{above}(L O, R O)=g(\delta) \cdot \operatorname{height}\left(y_{L O}\right)
$$

\section{MODEL COMPARISON}

In the previous section, we have presented the AVS model by Regier and Carlson (2001) and proposed the rAVS model, since the AVS model conflicts with recent empirical findings regarding the direction of the attentional shift (Burigo \& Knoeferle, 2015; Franconeri et al., 2012; Roth \& Franconeri, 2012). But how does the rAVS model perform in comparison to the AVS model?

Regier and Carlson (2001) conducted seven experiments and showed that the AVS model was able to account for all empirical data from these experiments. We evaluated the rAVS model on the same data set to assess its performance. Before we present the results, we briefly introduce the method that we applied.

\subsection{Method}

To assess the two models, we fitted them to the data Regier and Carlson (2001) used to evaluate the AVS model: the data from seven acceptability rating tasks conducted by Regier and Carlson (2001). These data consist of acceptability ratings for 337 locations of the LO above 10 different types of ROs. ${ }^{4}$ We fitted both models to these data by minimizing the Root Mean Square Error (RMSE). To this end, we used a method known as simulated annealing, a variant of the Metropolis algorithm (Metropolis, Rosenbluth, Rosenbluth, Teller, \& Teller, 1953). This method estimates the parameters of the model in order to minimize the RMSE and has the advantage to not get stuck in local minima. The found RMSE gives us a Goodness-Of-Fit (GOF) value.

\footnotetext{
${ }^{3}$ Therefore, the rAVS model does not need to compute a vector sum nor does it rely on an underlying attentional distribution and thus has a lower computational complexity. This lower computational complexity, however, originates from the simplification of the LO. Accordingly, these considerations are also only valid for simplified LOs.

${ }^{4}$ We thank Terry Regier and Laura Carlson for sharing these data.
} 
Since more complex models might obtain a better GOF value just because of their complexity (Pitt \& Myung, 2002), we also applied a cross-validation method that takes model complexity into account: the simple hold-out (SHO) method described in Schultheis, Singhaniya, and Chaplot (2013). Schultheis et al. (2013) showed that this method performs very well in comparison to other model comparison methods. In the SHO method, the data set is split into a training and a test set. Model parameters are estimated on the training set and used to compute a prediction error (RMSE) on the test set. This is done several times with different, random splits of the data. The median of the prediction error is the final outcome of the SHO method.

The results presented here were computed with 101 iterations of the SHO method. In each iteration $70 \%$ of the data was used as training data and $30 \%$ was used as test data. Moreover, we computed $95 \%$ confidence intervals of the SHO median by using 100,000 bootstrap samples with the help of the boot package for R (Canty \& Ripley, 2015). Both models and the data fitting methods were implemented in $\mathrm{C}++$ with the help of the Computational Geometry Algorithms Library (CGAL, Computational Geometry Algorithms Library, n.d.). The source code is available from Kluth (2016). We constrained the range of the model parameters for both the GOF and the SHO computation in the following way:

$$
\begin{aligned}
\frac{-1}{45} & \leq \text { slope } \leq 0 \\
0.7 & \leq \text { intercept } \leq 1.3 \\
0 & \leq \text { highgain } \leq 10 \\
0 & <\lambda \leq 5 \\
0 & <\alpha \leq 5
\end{aligned}
$$

\subsection{Results}

Figure 3 shows the GOF and SHO results for fitting both models to all data from Regier and Carlson (2001). The model parameters for the plotted GOFs can be found in Table 1. First of all, both models are able to account for the data very closely as is evident from the overall low RMSE. A RMSE of 0 would mean that both models can produce the exact empirical data. The theoretically worst possible RMSE of 9 means that model and data are maximally different. This worst value is 9 because Regier and Carlson (2001) used a rating scale from 0 to 9 . Consider rating data where humans rated all LOs with a 9. The model, however, computes only 0 s. This would then result in the worst possible RMSE of 9.

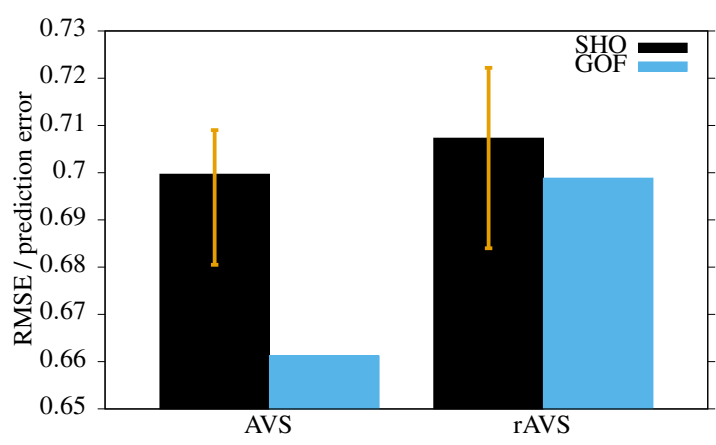

Figure 3: GOF and SHO results for the AVS and the rAVS model for fitting all data from Regier and Carlson (2001). Error bars show $95 \%$ confidence intervals computed with 100,000 bootstrap samples.

Table 1: Values of the model parameters and RMSE to achieve the GOF shown in Figure 3. The $\lambda$ parameter of the rAVS model does not change the output of the rAVS model, see footnote 3 .

\begin{tabular}{lcc} 
& AVS & rAVS \\
\hline slope & -0.005 & -0.004 \\
intercept & 0.973 & 0.943 \\
highgain & 0.083 & 7.497 \\
$\lambda$ & 0.189 & $(1.221)$ \\
$\alpha$ & - & 0.322 \\
\hline RMSE & 0.661 & 0.699
\end{tabular}

The rAVS model has a slightly worse GOF value, but the value is still very low and only shows a small difference $(<0.04)$ to the GOF value of the AVS model. In light of the problems with using GOF measures discussed by Pitt and Myung (2002) and Roberts and Pashler (2000), this small difference in the GOF remains inconclusive. Moreover, the GOF values itself change slightly with each new estimation due to the random nature of the parameter estimation method. The most important conclusion one can draw from the GOF values is whether the models are able to fit the data at all. Assessing the relative performance of more than one model solely with their GOF, however, should be done very carefully.

The SHO values, on the other hand, are suitable to compare the performance of two or more models. In our case, both models obtain similar SHO values with overlapping confidence intervals for the SHO values. That is, both models perform equally well and cannot be distinguished on these data. Accordingly, both directions of the attentional shift are equally well supported by these model simulations. 


\subsection{Discussion}

Although our model simulations do not result in the support of one of the two shifts in question, they rise the question to which degree the attentional shift from the RO to the LO as theorized by Logan (1995) and Logan and Sadler (1996) is the only shift that is implicated in the processing of spatial relations. The results from Burigo and Knoeferle (2015) suggest that humans perform both shifts, but that the shift from the LO to the RO alone (as in the rAVS model) can be enough to apprehend the spatial relation between the objects. The shift back (from the RO to the LO) could be a way to double-check the goodness-of-fit of the spatial preposition. Our results support this by showing that the rAVS model - that assumes only the shift from the LO to the RO - can account for the data from Regier and Carlson (2001).

\section{CONCLUSION}

We proposed a new cognitive model for spatial language understanding: the rAVS model. This model is based on the AVS model by Regier and Carlson (2001) but integrates recent psycholinguistic and neuroscientific findings (Burigo \& Knoeferle, 2015; Franconeri et al., 2012; Roth \& Franconeri, 2012) that conflict with the assumption of the direction of the attentional shift in the AVS model. In the AVS model, attention shifts from the RO to the LO; in the rAVS model, attention shifts from the LO to the RO. We assessed both models using the data from Regier and Carlson (2001) and found that both models perform equally well. Accordingly, our model simulations do not favor any of the two models and thus, do also not favor any of the two directionalities of the attentional shift.

Theoretical Contribution Regier and Carlson (2001) developed the AVS model with the goal to identify possible nonlinguistic mechanisms that underlie spatial term rating. To this end, they implemented two independent observations in the AVS model: First, the importance of attention to understand spatial relations and second, the neuronal representation of a motor movement as a vector sum. So, the main goal of the AVS model was not to examine the direction of the shift of attention but rather to describe linguistic processes with nonlinguistic mechanisms.

Although the focus of the AVS model was not on the direction of the attentional shift, the model implies a shift from the RO to the LO. Regier and Carlson
(2001) motivated the use of a vector sum because it seems to be a widely used representation of direction in the brain. Georgopoulos, Schwartz, and Kettner (1986) found that the direction of an arm movement of a rhesus monkey can be predicted by a vector sum of orientation tuned neurons. Lee, Rohrer, and Sparks (1988) found a similar representation for saccadic eye movements. Eye movements (overt attention) are motor movements that are closely connected to covert visual attention: "Many studies have investigated the interaction of overt and covert attention, and the order in which they are deployed. The consensus is that covert attention precedes eye movements [...]." (Carrasco, 2011, p. 1487) Although the authors of the AVS model do not explicitly speak about which movement the vector sum in their model represents nor do they clearly specify the kind of attention in the model, it seems reasonable to interpret the direction of the vector sum in the AVS model as the direction of a shift of attention that goes from the RO to the LO.

Our aim is to implement the most recent findings of attentional mechanisms into the AVS model. To this end, we designed the rAVS model as similar as possible to the AVS model. So, the rAVS model follows the same basic concepts whilst it integrates the most recent findings. We do not claim that the nonlinguistic mechanisms proposed in the AVS model do not happen - rather, we propose an alternate way how they might take place. Keeping the same basic concepts as the AVS model, the rAVS model accounts for the same data equally well - and also for the recent empirical findings regarding the direction of the attentional shift.

Model Complexity Due to the simplification of the LO as a single dot, the vector sum in the rAVS model consists of only one vector, i.e., there is no population of vectors to be processed. This drastically reduces the time needed for computation.

The attentional distribution in combination with the vector sum are giving the AVS model a high amount of flexibility (the flexibility of a model is strongly connected to its complexity, Pitt \& Myung, 2002). While a cognitive model should be flexible enough to account for individual differences, it should not be too flexible. A model that is too flexible could otherwise fit data that humans would never generate (see Roberts \& Pashler, 2000, for a thorough discussion of this issue). ${ }^{5}$

The flexibility of the AVS model (stemming from the complex interplay of the attentional distribution

\footnotetext{
${ }^{5}$ However, it could be that the human cognitive processes can only be described with a flexible model (simply because they are complex).
} 
and the vector sum) makes it hard to analyze the AVS model: It is often not easy to determine the influence of, say, the relative distance of the LO to the RO on the behavior of the model. This is particular true if one considers different values of the model parameters. The rAVS model, on the other hand, has clear formulations for the relative distance that do not change in their qualitative behavior with different sets of parameters. Still, the rAVS model shows the same performance as the AVS model on the data from Regier and Carlson (2001).

Note that the lower computational complexity of the rAVS model arises from the simplification of the LO. Conceptually, the rAVS model also computes an attentional vector sum that points from the LO to the RO. Thus, the discussion of the model flexibility is only valid for rating data that was collected with a simplified LO (as the data from Regier \& Carlson, 2001). A more comprehensive model of spatial language should also represent the LO in more detail (see Future Work). It remains to be seen whether or not a model with a single vector can also account for the processing of spatial relations when the LO has a mass.

\subsection{Future Work}

Modeling Both Shifts The success of both the rAVS model and the AVS model support the existence of both directionalities of the attentional shift. It might well be that people shift their attention in both directions during the processing of spatial relations depending on the task and the linguistic input. Accordingly, a model that implements both attentional shifts might fit more data than the AVS or the rAVS model. ${ }^{6}$

It might be interesting to investigate this possibility by creating a model that allows both shifts of attention. Such model should be applicable to more types of experimental data than the AVS model and the rAVS model (which both can only account for acceptability rating data). In particular, the model with both shifts should also specify when in time what type of attentional shift occurs and how long the computation takes. This model could then be fitted to a greater range of data, like real-time eye movement data from visual world studies (e.g., Burigo \& Knoeferle, 2015) or reaction time data (e.g., Roth \& Franconeri, 2012). Modeling different tasks would give more insight into the role of the attentional shift.

\footnotetext{
${ }^{6}$ We thank an anonymous reviewer for suggesting this
}

Modeling the LO The reason for the lower computational complexity of the rAVS model is the simplification of the LO as a single point (this was done to keep the rAVS model as close as possible to the AVS model). There is evidence, however, that geometric features of the LO also affect acceptability ratings (Burigo, 2008; Burigo, Coventry, Cangelosi, \& Lynott, in press; Burigo \& Sacchi, 2013). A comprehensive model of spatial language thus should also model the LO in more detail. Accordingly, we are planning to extend the representation of the LO in the rAVS model by giving a mass to it. This would give us the opportunity to see first how the rAVS model deals with the situation where the computation of a vector sum is necessary to determine the angular deviation. Second, this changes the role of the attentional distribution in the rAVS model.

We are also planning to change the use of the height component in the rAVS model. At the moment, the rAVS model applies the same computation as the AVS model for the height component: the $y$ coordinate of the LO is compared relative to the top of the RO (see Fig. 1(d)). In the rAVS model, the attentional focus is located on the LO. So, it would be more consistent if the location of the LO is taken as the baseline for the comparison with the location of the RO. Thus, we want to reverse the computation of the height component such that the grazing line lies on the bottom of the LO.

Model Distinction To tease apart the two models and evaluate the accuracy of their predictions, we are currently analyzing the models with an algorithm called Parameter Space Partitioning (PSP) proposed by Kim, Navarro, Pitt, and Myung (2004); Pitt, Kim, Navarro, and Myung (2006). The PSP algorithm is a Markov chain Monte Carlo (MCMC) based method and searches in the parameter space of the models for regions of patterns that are qualitatively different. First results confirm the high flexibility of the AVS model (i.e., the AVS model is able to generate many patterns that are qualitatively different by using different sets of parameters). The rAVS model, however, generates fewer patterns with a qualitative difference.

The PSP analysis seems to confirm that the two models make different predictions for the displays under consideration. We are planning an empirical rating study that tests these different predictions. With the data collected in this study, we should be able to distinguish which model makes more accurate predictions.

We are also planning to further compare the two models with both versions of the parametric bootstrap crossfitting method (Navarro, Pitt, \& Myung, 2004; 
Wagenmakers, Ratcliff, Gomez, \& Iverson, 2004).

Functionality The AVS model does not account for any effects of the functionality of objects on spatial language comprehension, although there is evidence that - beside purely geometric effects - functional interactions between objects also affect the use of spatial prepositions (Carlson, Regier, Lopez, \& Corrigan, 2006; Carlson-Radvansky, Covey, \& Lattanzi, 1999; Coventry \& Garrod, 2004; Coventry et al., 2010; Coventry, Prat Sala, \& Richards, 2001; Hörberg, 2008).

For instance, Carlson-Radvansky et al. (1999) conducted an object placement task, where participants had to place a toothpaste tube above a toothbrush. They showed that the toothpaste tube was not placed above the center-of-mass of the toothbrush, but rather above the bristles of the toothbrush - that is, at the location where both objects can functionally interact. Objects with a smaller amount of functional interaction (here, a tube of oil paint) were placed more above the center-of-mass of the toothbrush instead over the bristles.

Despite this evidence, the AVS model (and thus also our rAVS model) only considers geometric representations of the RO and the LO. For the AVS model, however, a range of extensions that integrate functionality were already proposed (Carlson et al., 2006; Kluth \& Schultheis, 2014). Since the rAVS model is designed to be as similar as possible to the AVS model, these functional extensions might also be applicable for the rAVS model.

Implementing the Models in Artificial Systems In order to implement these models into artificial systems, additional steps are necessary. The models were designed to model spatial language understanding. So, the models produce an acceptability rating given a RO, a LO, and a preposition. As part of an artificial system that interprets spatial language, the models can be used straightforwardly: Given a spatial utterance and a visual scene, the models can be used to compute acceptability ratings for all points around the RO (i.e., a spatial template). The artificial system then starts the search for the LO at the point with the highest rating.

To generate spatial language with the help of these models, one could imagine the following steps: Compute the acceptability ratings of different spatial prepositions (e.g., above, below, to the left of, in front of, ...) and subsequently pick the one with the highest rating.

In conclusion, we proposed a modified version of the AVS model: the rAVS model. The rAVS model accounts for the same empirical data as the AVS model while integrating additional recent findings regarding the direction of the attentional shift that conflict with the assumptions of the AVS model.

\section{ACKNOWLEDGMENTS}

This research was supported by the Cluster of Excellence Cognitive Interaction Technology 'CITEC' (EXC 277) at Bielefeld University, which is funded by the German Research Foundation (DFG). The authors would also like to thank two anonymous reviewers for their useful comments and suggestions.

\section{References}

Burigo, M. (2008). On the role of informativeness in spatial language comprehension (Unpublished doctoral dissertation). School of Psychology, University of Plymouth.

Burigo, M., Coventry, K. R., Cangelosi, A., \& Lynott, D. (in press). Spatial Language and Converseness. Quarterly Journal of Experimental Psychology.

Burigo, M., \& Knoeferle, P. (2015). Visual attention during spatial language comprehension. PloS ONE, 1O(1), e0115758. doi: 10.1371/journal.pone. 0115758

Burigo, M., \& Sacchi, S. (2013). Object orientation affects spatial language comprehension. Cognitive Science, 37(8), 1471-1492.

Cangelosi, A., Coventry, K. R., Rajapakse, R., Joyce, D., Bacon, A., Richards, L., \& Newstead, S. N. (2005). Grounding language in perception: A connectionist model of spatial terms and vague quantifiers. Progress in Neural Processing, 16, 47.

Canty, A., \& Ripley, B. (2015). boot: Bootstrap R (SPlus) Functions [Computer software manual]. (R package version 1.3-15)

Carlson, L. A., \& Logan, G. D. (2005). Attention and spatial language. In L. Itti, G. Rees, \& J. K. Tsotsos (Eds.), Neurobiology of Attention (pp. 330-336). Elsevier.

Carlson, L. A., Regier, T., Lopez, W., \& Corrigan, B. (2006). Attention unites form and function in spatial language. Spatial Cognition and Computation, 6(4), 295-308.

Carlson-Radvansky, L. A., Covey, E. S., \& Lattanzi, K. M. (1999). What effects on where: Func- 
tional influences on spatial relations. Psychological Science, 10(6), 516-521.

Carrasco, M. (2011). Visual attention: The past 25 years. Vision Research, 51(13), 1484-1525.

Coventry, K. R., \& Garrod, S. C. (2004). Saying, seeing, and acting: The psychological semantics of spatial prepositions. Hove and New York: Psychology Press, Taylor and Francis.

Coventry, K. R., Lynott, D., Cangelosi, A., Monrouxe, L., Joyce, D., \& Richardson, D. C. (2010). Spatial language, visual attention, and perceptual simulation. Brain and Language, 112(3), 202-213.

Coventry, K. R., Prat Sala, M., \& Richards, L. (2001). The interplay between geometry and function in the comprehension of over, under, above, and below. Journal of Memory and Language, 44(3), 376-398.

Franconeri, S. L., Scimeca, J. M., Roth, J. C., Helseth, S. A., \& Kahn, L. E. (2012). Flexible visual processing of spatial relationships. Cognition, 122(2), 210-227.

Gapp, K.-P. (1995). An empirically validated model for computing spatial relations. In I. Wachsmuth, C.-R. Rollinger, \& W. Brauer (Eds.), KI-95: Advances in Artificial Intelligence (Vol. 981, p. 245-256). Springer Berlin Heidelberg. doi: 10.1007/3-540-60343-3_41

Georgopoulos, A. P., Schwartz, A. B., \& Kettner, R. E. (1986). Neuronal Population Coding of Movement Direction. Science, 233, 14161419.

Hörberg, T. (2008). Influences of form and function on the acceptability of projective prepositions in swedish. Spatial Cognition \& Computation, 8(3), 193-218.

Kelleher, J. D., Kruijff, G.-J. M., \& Costello, F. J. (2006). Proximity in context: an empirically grounded computational model of proximity for processing topological spatial expressions. In Proceedings of the 21st International Conference on Computational Linguistics and the 44th annual meeting of the Association for Computational Linguistics (pp. 745-752).

Kim, W., Navarro, D. J., Pitt, M. A., \& Myung, I. J. (2004). An MCMC-based method of comparing connectionist models in cognitive science. Advances in Neural Information Processing Systems, 16, 937-944.

Kluth, T. (2016). A C++ Implementation of the reversed Attentional Vector Sum (rAVS) model. Bielefeld University. doi: 10.4119/unibi/2900103

Kluth, T., \& Schultheis, H. (2014). Attentional distribution and spatial language. In C. Freksa, B. Nebel, M. Hegarty, \& T. Barkowsky (Eds.), Spatial Cognition IX (Vol. 8684, pp. 7691). Springer International Publishing. doi: 10.1007/978-3-319-11215-2_6

Lee, C., Rohrer, W. H., \& Sparks, D. L. (1988). Population coding of saccadic eye movements by neurons in the superior colliculus. Nature, 332, 357-360.

Logan, G. D. (1994). Spatial attention and the apprehension of spatial relations. Journal of Experimental Psychology: Human Perception and Performance, 20(5), 1015.

Logan, G. D. (1995). Linguistic and conceptual control of visual spatial attention. Cognitive Psychology, 28(2), 103-174.

Logan, G. D., \& Sadler, D. D. (1996). A computational analysis of the apprehension of spatial relations. In P. Bloom, M. A. Peterson, L. Nadel, \& M. F. Garrett (Eds.), Language and Space (pp. 493-530). The MIT Press.

Metropolis, N., Rosenbluth, A. W., Rosenbluth, M. N., Teller, A. H., \& Teller, E. (1953). Equation of state calculations by fast computing machines. The journal of chemical physics, 21(6), 1087-1092.

Navarro, D. J., Pitt, M. A., \& Myung, I. J. (2004). Assessing the distinguishability of models and the informativeness of data. Cognitive Psychology, 49(1), 47-84.

Pitt, M. A., Kim, W., Navarro, D. J., \& Myung, J. I. (2006). Global model analysis by parameter space partitioning. Psychological Review, 113(1), 57.

Pitt, M. A., \& Myung, I. J. (2002). When a good fit can be bad. Trends in cognitive sciences, 6(10), $421-425$.

Regier, T., \& Carlson, L. A. (2001). Grounding spatial language in perception: An empirical and computational investigation. Journal of Experimental Psychology: General, 130(2), 273298.

Richter, M., Lins, J., Schneegans, S., Sandamirskaya, Y., \& Schöner, G. (2014). Autonomous Neural Dynamics to Test Hypotheses in a Model of Spatial Language. In P. Bello, M. Guarini, M. McShane, \& B. Scassellati (Eds.), Proceedings of the 36th Annual Conference of the Cognitive Science Society (pp. 2847-2852). Austin, TX: Cognitive Science Society. 
Roberts, S., \& Pashler, H. (2000). How persuasive is a good fit? A comment on theory testing. Psychological review, 107(2), 358-367.

Roth, J. C., \& Franconeri, S. L. (2012). Asymmetric coding of categorical spatial relations in both language and vision. Frontiers in Psychology, 3(464). doi: 10.3389/fpsyg.2012.00464

Schultheis, H., Singhaniya, A., \& Chaplot, D. S. (2013). Comparing model comparison methods. In Proceedings of the 35th Annual Conference of the Cognitive Science Society (pp. 1294 - 1299). Austin, TX: Cognitive Science Society.

CGAL, Computational Geometry Algorithms Library. (n.d.). (http://www.cgal.org)

Wagenmakers, E.-J., Ratcliff, R., Gomez, P., \& Iverson, G. J. (2004). Assessing model mimicry using the parametric bootstrap. Journal of Mathematical Psychology, 48(1), 28-50. 